University of Nebraska - Lincoln

DigitalCommons@University of Nebraska - Lincoln

Architectural Engineering -- Faculty Publications

Architectural Engineering and Construction,

Durham School of

2010

Optimum absorption and aperture parameters for realistic coupled volume spaces determined from computational analysis and subjective testing results

\author{
David T. Bradley \\ University of Nebraska-Lincoln, dabradley@vassar.edu \\ Lily M. Wang \\ University of Nebraska-Lincoln, Iwang4@unl.edu
}

Follow this and additional works at: https://digitalcommons.unl.edu/archengfacpub

Part of the Architectural Engineering Commons

Bradley, David T. and Wang, Lily M., "Optimum absorption and aperture parameters for realistic coupled volume spaces determined from computational analysis and subjective testing results" (2010).

Architectural Engineering -- Faculty Publications. 3.

https://digitalcommons.unl.edu/archengfacpub/3

This Article is brought to you for free and open access by the Architectural Engineering and Construction, Durham School of at DigitalCommons@University of Nebraska - Lincoln. It has been accepted for inclusion in Architectural Engineering -- Faculty Publications by an authorized administrator of DigitalCommons@University of Nebraska Lincoln. 


\title{
Optimum absorption and aperture parameters for realistic coupled volume spaces determined from computational analysis and subjective testing results
}

\author{
David T. Bradley ${ }^{\text {a) }}$ and Lily M. Wang \\ Architectural Engineering Program, Peter Kiewit Institute, University of Nebraska-Lincoln, Omaha, \\ Nebraska 68182-0681
}

(Received 16 June 2009; revised 22 October 2009; accepted 3 November 2009)

\begin{abstract}
This project utilizes computational modeling to study the effects of varying two architectural parameters, absorption ratio and aperture size, in a realistic coupled volume concert hall. Coupled volumes have been shown to exhibit non-exponential sound energy decay profiles, referred to as double slope effect. A number of objective metrics (T30/T15, LDT/T10, decay ratio, and $\Delta L$ ) have been used to quantify the double slope effect of the profiles generated in the virtual hall. T30/T15 and LDT/T10 showed similar trends across all hall configurations, indicating decreasing double slope effect with increasing coupled volume absorption ratio for each aperture size, and producing highest values at a specific aperture size for each absorption ratio. Generally, LDT/T10 provides finer resolution than T30/T15 when analyzing the decay profiles in this study. Results from the two metrics derived from Bayesian analysis, decay ratio and $\Delta L$, seem less consistent. Subjective testing has also been conducted to determine the effect of varying the two architectural parameters in the hall, and multidimensional scaling analysis shows that, in general, listener preference is inversely proportional to the level of double slope effect, with the highest levels of preference occurring at low and medium levels of double slope effect. Recommended design guidelines for coupled volume halls are provided based on these computational and subjective results.
\end{abstract}

(C) 2010 Acoustical Society of America. [DOI: 10.1121/1.3268604]

PACS number(s): 43.55.Br, 43.55.Ka, 43.55.Hy, 43.55.Fw [ADP] Pages: 223-232

\section{INTRODUCTION}

Spaces exhibiting acoustically coupled volumes are typically composed of a highly reverberant secondary room connected to a main room via an acoustically transparent aperture. This geometrical configuration is of particular interest due to the potential for the creation of temporally disparate decay rates in the main room. This so-called non-exponential decay is atypical in single volume spaces, and can lead to unique acoustic conditions. In particular, coupled volume systems can be designed to produce a non-exponential decay that exhibits a steep initial decay followed by a long reverberant tail. This type of decay is assumed to produce high perceived levels of both clarity and reverberance, qualities that are typically desirable in performing arts spaces. The current work focuses on determining the acoustic effect of architectural parameters such as the amount of absorption in the coupled spaces and the size of the aperture opening between them. Particular attention is given to studying how the sound energy decay within coupled volume spaces varies in response to changing architectural parameters, and how human listeners respond to these variations. This research is an extension of previous work in which the authors examined coupled volumes consisting of two rectangular boxes connected to one another. ${ }^{1}$ The current study explores a more complicated and realistic geometry such as those found in

\footnotetext{
a) Author to whom correspondence should be addressed. Present address: Physics+Astronomy Department, Vassar College, Poughkeepsie, New York 12604-0745. Electronic mail: dabradley@vassar.edu
}

existing coupled volume performing arts spaces. Furthermore, psychoacoustics testing has been conducted to determine the preference of listeners for various decay profiles from the virtual coupled volume hall. The results are used to provide a design rubric for implementing coupled volume geometries in auditoria.

A detailed theoretical development of the sound energy decay in coupled volumes can be found in literature. ${ }^{1-6}$ To summarize, for the particular case when a highly reverberant secondary volume is coupled with a less reverberant main volume, sound energy from the secondary space can feed back into the main space through the aperture opening between them. When the sound source and receiver are located in the main volume, this delayed feedback of sound energy can result in a decay profile with more than one slope. Decay profiles are typically plotted on a logarithmic scale, such that a single slope decay depicts an exponential decay. A double slope decay is referred to as non-exponential; a representative decay profile is shown in Fig. 1. This double slope decay can be represented by the two linear functions of time shown as solid and dashed bold lines in Fig. 1, such that ${ }^{3}$

$$
\begin{aligned}
& L_{1}(t)=-\left(\frac{60}{T_{1}}\right) t, \\
& L_{2}(t)=-\left(\frac{60}{T_{2}}\right) t-10 \log \left(\frac{A_{1 S} A_{2 S}}{S^{2}}\right),
\end{aligned}
$$

where $A_{i s}=A_{i}+S, A_{i}$ is the equivalent absorption area of the $i$ th space (inclusive of the aperture opening area), $S$ is the 


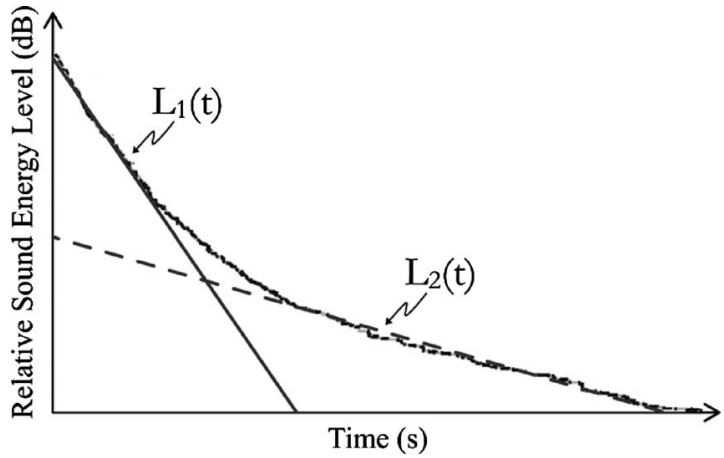

FIG. 1. A representative decay outline exhibiting a double slope, where $L_{1}(t)$ is shown by the solid line and $L_{2}(t)$ by the dashed line.

surface area of the aperture opening, and $T_{i}$ is the decay time for room $i$ alone. Equations (1) and (2) are in the form of $y=-m x+b$. This allows for the decay slope of the main volume, when coupled, to be analyzed based on its two temporal components, early decay $\left(L_{1}(t)\right)$ and late decay $\left(L_{2}(t)\right)$. Each portion of the decay is given by a line with a slope and $y$-intercept that can be calculated from architectural parameters of the coupled volume system. ${ }^{1}$ Double slope effect (DSE) will be used in this paper to refer to the phenomenon when the second slope is larger than the first, and dominates the decay profile during the late portion (as shown in Fig. 1).

For this investigation, a coupled volume concert hall was designed based on an amalgamation of characteristics from existing coupled volume halls. The design of this imagined hall was then implemented as a computer model using computer aided drafting (CAD) software. An acoustics computer modeling program, ODEON (version 6.5), was used to predict room impulse responses (RIRs) for several configurations of the virtual hall computer model. The RIRs were studied to determine the effect of changes in the architectural parameters on sound decay in the virtual hall. Then, the response of listeners to the sound fields created in the computer models was determined from subjective testing and subsequent statistical analysis.

\section{PREVIOUS RESEARCH}

Several investigations have been conducted on the acoustics of coupled volume systems, using both physical and computational models based on geometrical acoustics. $^{7-14}$ Of particular note, Nijs et al. ${ }^{9}$ compared scale and computational model results of coupled volume spaces and listed several best practice techniques for increasing the fidelity of the computational simulations. These techniques have been implemented in the computational work described in this paper. Also, Bradley and Wang ${ }^{14}$ validated the use of ODEON (version 6.5) for such studies by comparing simulated and measured impulse responses in a coupled volume concert hall and finding good correlations in the data.

In some of this previous work, metrics have been proposed for quantifying DSE in coupled volume halls. Harrison and Madaras ${ }^{10}$ used the ratio T30/T15 to give the relationship between two portions of the reverberant decay in the main room. T30 is defined as the decay time with sound pressure level (SPL) limits of -5 and $-35 \mathrm{~dB}$ in the energy decay function, multiplied by a factor of 2. ${ }^{15}$ Similarly, T15 is given as the decay time from -5 to $-20 \mathrm{~dB}$, multiplied by a factor of 4. T30/T15 gives the relationship between the overall decay curve and the early portion of the decay, therefore providing an indication of the relationship between the two slopes in a non-exponential decay. Harrison and Madaras $^{10}$ found that T30/T15 values were highest for smaller aperture opening sizes, and increased with larger coupled volume sizes. Ermann ${ }^{13}$ used a similar ratio of T60/ T15 in his work, wherein he studied the effect of aperture size and coupled volume absorption on DSE using numerical and CATT ACOUSTIC (version 8.0b) models of an imagined coupled volume concert hall. His work showed that having an average absorption coefficient $(\bar{\alpha})$ in the coupled space less than $0.02(\bar{\alpha}<0.02)$ produced significantly higher T60/ $\mathrm{T} 15$ values than $0.02<\bar{\alpha}<0.05$. Also T60/T15 values were largest when the aperture size was relatively small (1\% of the total main volume surface area).

While these DSE quantifiers, T30/T15 and T60/T15, can describe general trends in DSE, they do not offer a method for differentiating between different double slope profiles. Particularly, specific T30/T15 and T60/T15 values can be used to describe several different double slope profiles. Furthermore, the decay measures in the numerator and denominator of each ratio may not be a good linear fit of the decay curve over the range covered, which can misrepresent the two slopes in the decay. The two decay measure ranges also overlap, which can obfuscate the double slope nature of the decay. These ambiguities compromise the effectiveness of these descriptors as quantifiers of DSE. Bradley and Wang ${ }^{1}$ tested two quantifiers that can theoretically identify DSE profiles more uniquely: the decay ratio $\left(T_{2} / T_{1}\right)$ and $\Delta L . \Delta L$ is defined as the difference in decibels between the two $y$-intercepts in the DSE profile (referred to as $\Delta \mathrm{dB}$ in previous studies), calculated from data produced by a Bayesian analysis algorithm developed by Xiang et al. ${ }^{5,6,16}$ In this investigation, decay ratio and $\Delta L$ showed trends similar to T30/T15, except for cases with the largest coupled volume size in which the results were harder to interpret. ${ }^{1}$

In another study, Bradley and Wang ${ }^{17}$ analyzed computational models of an existing coupled volume concert hall, and compared the DSE quantifiers listed above as well as a newly proposed quantifier, late decay time/early decay time (LDT/EDT). EDT is defined as the decay time from 0 to -10 $\mathrm{dB}$, multiplied by a factor of $6 .{ }^{15}$ LDT was proposed by the authors as a measure of the late portion of the decay. This decay measure is defined as the decay time with SPL limits of -25 and $-35 \mathrm{~dB}$ in the energy decay function, multiplied by a factor of 6 . This SPL range was chosen to correspond with the bottom of the established T30 range, which ensures that the decay values are above the noise floor for measurements taken according to ISO 3382-1. ${ }^{15}$ The LDT/EDT ratio, unlike T30/T15, takes the ratio of two distinct portions of the decay, and more closely approximates the ratio of the two slopes found in a double slope decay than ratios that cover overlapping decay ranges. This distinction makes LDT/EDT likely to quantify DSE more accurately than T30/T15 or T60/ T15. Indeed, Bradley and Wang found that LDT/EDT seemed to differentiate DSE with more resolution than the 
other ratios. The two metrics calculated from Bayesian analysis results, decay ratio and $\Delta L$, surprisingly did not indicate the presence of a double-sloped decay curve in this study, though.

Upon subsequent analysis, the authors have replaced LDT/EDT with a revised metric, LDT/T10, for the work described in this paper; T10 is defined as the decay time from -5 to $-15 \mathrm{~dB}$, multiplied by a factor of 6 . One can expect that LDT/T10 should resolve to a value of 1 when the decay curve is exponential. This may not necessarily occur with LDT/EDT since EDT is much more influenced by early reflection patterns than T10.

None of the metrics introduced so far has been previously linked to subjective preference in a suitable manner. Some previous subjective studies have focused on perception of exponential decays versus non-exponential decays generated by splicing together signals from computer-simulated single-sloped decays. ${ }^{18,19}$ Picard's results, in particular, showed that subjects could more easily perceive a difference between the decays when the $y$-intercepts of the two slopes of the decay were minimized (i.e., smaller $\Delta L) .{ }^{19}$ Additionally, subjects were more likely to recognize a difference between two non-exponential decay curves when the difference between the first and second slopes in the non-exponential decay was increased (i.e., larger decay ratio $T_{2} / T_{1}$ ).

Bradley and Wang ${ }^{1}$ conducted a subjective study using a range of decay curves generated from simplistic representations of coupled volume performance spaces, created from two rectangular boxes connected to one another. This study sought to determine if varying degrees of double slope effect were perceived to have greater or less reverberation and/or clarity. Results showed that increasing the coupled volume size and the aperture size correlated with higher perceived reverberance, which matched objective T30/T15 results as well as possibly higher decay ratios and lower $\Delta L$ values. No statistically significant results on perceived clarity were found, though, in part due to the simplistic geometry used. Note that subjective preference between the decay curves was not investigated.

More recently, Ermann ${ }^{20}$ conducted subjective testing using exponential and non-exponential decays created from computational models of coupled volume spaces in an attempt to determine preference of double slope versus single slope decays. The results from this investigation agreed with Picard's results, showing that difference perception increased as the difference between the two slopes in the nonexponential decays was increased. However, no conclusion on preference could be made, as the sample size was small and many subjects could not correctly identify when two identical stimuli were presented in a paired comparison, indicating a problem with the reliability of the testing.

The research described in this paper advances the field of coupled volume room design by providing statistically significant data on subjective preference concerning coupled volume decays while linking that preference to objective measures of DSE. A computational model of a realistic coupled volume geometry has been systematically varied in terms of the absorption in the coupled spaces and the aperture opening between the spaces. The resulting room impulse
TABLE I. Architectural parameters and calculated volume ratio of the complex coupled volume system concert hall.

\begin{tabular}{lc}
\hline \hline Volume $\left(\mathrm{m}^{3}\right)$ [main] & 22145 \\
Total surface area $\left(\mathrm{m}^{2}\right)$ [main] & 5140 \\
Average absorption coefficient $\alpha$ [main] & 0.39 \\
Equivalent absorption area $S \alpha$ main] & 2005 \\
Volume $\left(\mathrm{m}^{3}\right)$ [coupled] & 10444 \\
Total surface area $\left(\mathrm{m}^{2}\right)$ [coupled] & 5075 \\
Volume ratio & $47 \%$ \\
\hline
\end{tabular}

responses have been analyzed for the degree of DSE, according to the metrics discussed above. Auralizations of these impulse responses have then been generated and used in subjective testing to determine relative preference of the decay curves. This work provides a comprehensive comparative analysis of objective and subjective data from these types of spaces, concluding with architectural design recommendations for achieving maximum subjective preference.

\section{COMPUTATIONAL ANALYSIS}

\section{A. Methodology}

The design for the virtual coupled volume concert hall in this study was based on a combination of architectural features from several existing concert halls with coupled volumes incorporated in their design, including Culture and Congress Centre Concert Hall (Lucerne, Switzerland), Theatres on the Bay Concert Hall (Esplanade, Singapore), McDermott Concert Hall (Dallas, TX), and Symphony Hall (Birmingham, UK). The pertinent architectural parameters and the volume ratio, calculated by dividing the main volume size by the secondary volume size, of the virtual hall are listed in Table I. Since previous work by the authors found that larger coupled volume sizes were more likely to generate double slope effect across different combinations of absorption characteristics and aperture opening sizes, ${ }^{1}$ a larger volume ratio from among the range in the existing halls surveyed was utilized in this investigation.

The shoebox-shaped main volume and the secondary volume of this virtual hall are connected through 236 aperture doors as shown in Fig. 2. The coupled volume extends the entire height of the hall on both sides of the stage and audience area, and is shown as light gray shading in Fig. 2. The doors are located on three levels in the hall, as illustrated by the dark gray shading in Fig. 2. The hall has an organ behind the stage, an acoustical canopy array hanging over the stage, and audience seating consisting of a main orchestra level and two stacked balcony levels.

Two architectural parameters were varied to create several configurations of the hall, as was done in previous work by the authors. ${ }^{1}$ The first architectural parameter is referred to in this paper as the absorption ratio, which quantifies the equivalent absorption area in the secondary volume as a portion of the equivalent absorption area in the main volume 


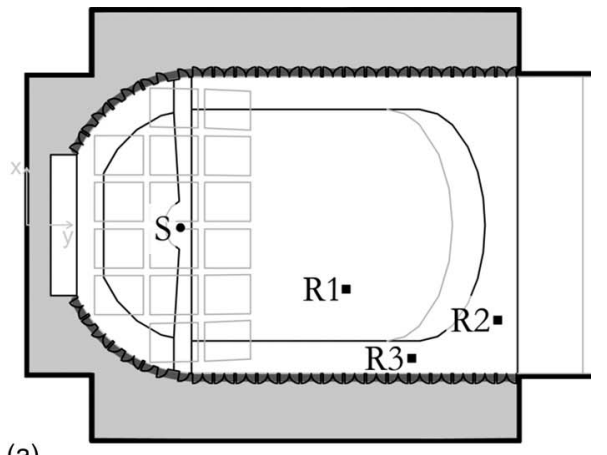

(a)

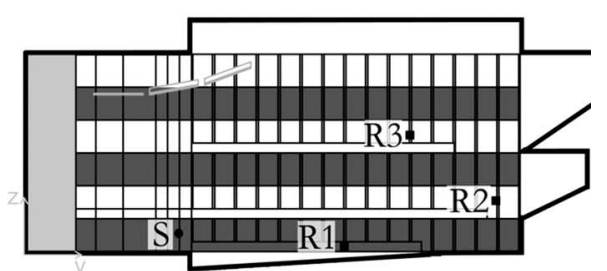

(b)

(c)

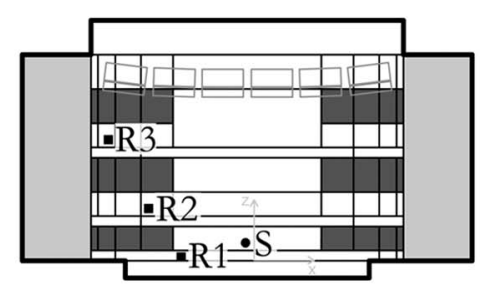

FIG. 2. (a) Plan view, (b) cross section, and (c) transverse cross section of the virtual hall with the secondary volume shaded in light gray and aperture doors in dark gray. Source position (s) is designated with a circle and receivers (R1-R3) are marked by squares.

$$
\text { absorption ratio }=\frac{\sum_{i}\left(\alpha_{i} S_{i}\right)_{\text {secondary }}}{\sum_{j}\left(\alpha_{j} S_{j}\right)_{\text {main }}},
$$

where the $i$ and $j$ summation counters represent the individual surfaces in the secondary and main volumes, respectively. The second parameter is aperture opening size expressed as a percentage of the total surface area of the main volume

$$
\text { aperture opening }=\frac{S_{\text {aperture }}}{\sum_{j}\left(S_{j}\right)_{\text {main }}} \times 100 \% .
$$

The aperture opening, as defined above, is normalized by the main volume surface area so that comparison across various coupled volume systems accounts for different volume sizes.

TABLE II. Four levels of absorption ratio used in the virtual hall configurations.

\begin{tabular}{cc}
\hline \hline Level & Absorption ratio \\
\hline 1 & 0.04 \\
2 & 0.08 \\
3 & 0.12 \\
4 & 0.16 \\
\hline
\end{tabular}

TABLE III. Seven levels of aperture size used in the virtual hall configurations.

\begin{tabular}{cc}
\hline \hline Level & \% of main volume surface area \\
\hline 0 & 0 \\
1 & 0.5 \\
2 & 1.0 \\
3 & 1.5 \\
4 & 3.0 \\
5 & 6.5 \\
6 & 10.0 \\
\hline \hline
\end{tabular}

Varying these two parameters can be expected to vary the shape of the double slope profile, as apparent from equations for decay ratio and $\Delta L$, presented by Bradley and Wang. ${ }^{1}$

The ranges of values for these architectural parameters were based on results from previous research and those typical in existing coupled volume halls. The parameter value ranges were also chosen to achieve a broad spectrum of DSE values. As such, four levels of absorption ratio were chosen, ranging from 0.04 to 0.16 , as shown in Table II. Additionally, seven levels of aperture size were chosen, ranging from $0 \%$ to $10 \%$ of the main volume surface area, as shown in Table III. The combination of these architectural parameter levels produced 25 configurations: 1 single volume system and 24 coupled volume systems.

Each configuration of the virtual hall was created as a CAD model and then imported into ODEON (version 6.5). These computer models were analyzed using 200000 rays from an omni-directional source with an impulse response length of $4500 \mathrm{~ms}$. Most surfaces were assigned a scattering coefficient of 0.3 ; surfaces likely to produce more nonspecular reflections, such as the canopy array and the organ, were given a scattering coefficient of 0.7 . These scattering coefficient values are based on the recommendations given for the ODEON program, ${ }^{21,22}$ and are similar to those used by the authors in a previous validation study. ${ }^{14}$

One source position and three receiver positions were studied in the models. The receivers were distributed evenly throughout one-half of the hall, taking advantage of symmetry. The source and receiver positions are shown as circles and squares, respectively, in Fig. 2. The coordinates of these positions are given in Table IV, with reference made to the origin shown in Fig. 2.

\section{B. Results}

An impulse response was generated for each of the 25 hall configurations for each source-receiver combination.

TABLE IV. Source and receiver coordinates for complex coupled volume system computer model configurations.

\begin{tabular}{lccc}
\hline \hline & $\begin{array}{c}x \\
(\mathrm{~m})\end{array}$ & $\begin{array}{c}Y \\
(\mathrm{~m})\end{array}$ & $\begin{array}{c}z \\
(\mathrm{~m})\end{array}$ \\
\hline Source & 14.5 & 0 & 2 \\
Receiver 1 & 30 & -6 & 0.6 \\
Receiver 2 & 44 & -8 & 4.4 \\
Receiver 3 & 35 & -12.3 & 10.5 \\
\hline \hline
\end{tabular}




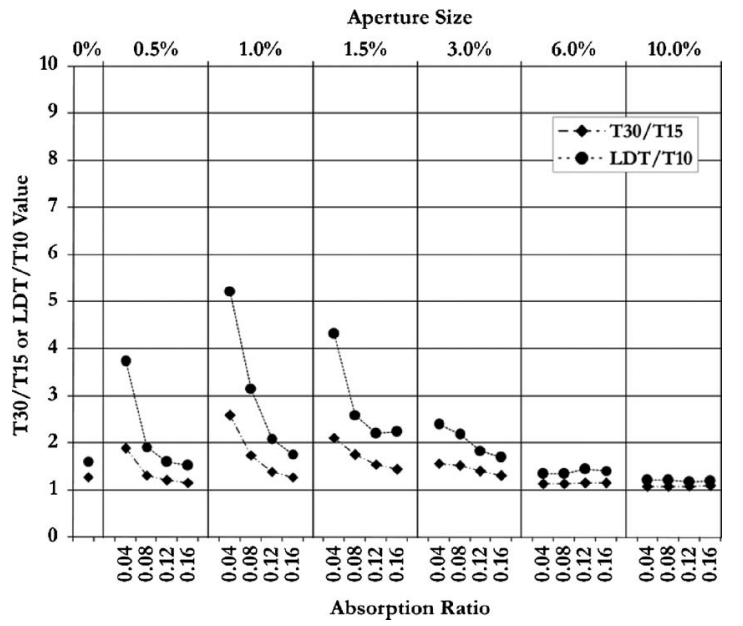

FIG. 3. T30/T15 and LDT/T10 data for the 25 virtual hall configurations

The impulse responses were exported from ODEON and analyzed to obtain several reverberation decay measures at the $1000 \mathrm{~Hz}$ octave band, including T15, T30, T10, and LDT. All decay measures were calculated from a least-squares fit line as prescribed in the ISO 3382-1 standard. ${ }^{15}$ The average difference between the receiver positions was taken across all configurations for each decay measure. The overall average difference across receiver positions for each decay measure was relatively small: less than $7 \%$. Therefore, only the results from receiver 1 will be discussed further in this paper.

Two DSE quantifiers, T30/T15 and LDT/T10, were calculated for the 25 virtual hall configurations, and are shown in Fig. 3. For all configurations, the T30/T15 and LDT/T10 data show similar trends for the varying architectural parameters. These DSE quantifiers decrease with increasing absorption ratio for each aperture size. The data across aperture size indicate that the two smaller absorption ratios $(0.04$ and 0.08 ) behave similarly, with maximum values for the DSE quantifiers found at aperture size $1.0 \%$. The two larger absorption ratios (0.12 and 0.16$)$ behave similarly, with the largest values found at aperture size $1.5 \%$. In general, the DSE values are much lower for the two larger aperture sizes $(6.0 \%$ and $10.0 \%)$. The DSE values for large aperture sizes closely match those for the single volume condition $(0 \%)$, indicating that the decays from these configurations behave like single slope decay.

The energy decay curves corroborate the conclusions drawn from the data for these two DSE quantifiers. Figure 4 shows a representative example of the decay curves for seven aperture size levels with the absorption ratio held constant at 0.04 . The single volume case $(0 \%)$ has a single slope decay, while the first four coupled configurations $(0.5 \%$, $1.0 \%, 1.5 \%$, and $3.0 \%$ ) exhibit a distinct double slope in their decay. However, the two largest aperture sizes $(6.0 \%$ and $10.0 \%$ ) have decays with less evident double slope character. In fact, the decay from aperture size $10.0 \%$ can be approximated as a single slope decay with a decay time much larger than the single volume case $(0 \%)$. In general, the larger aperture sizes produce decays with a single slope, as

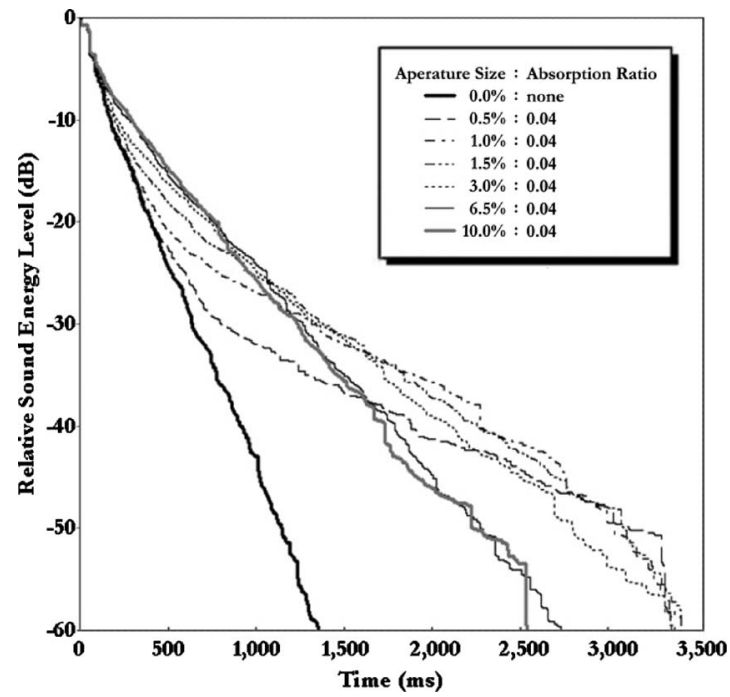

FIG. 4. Plot of energy decay curves for absorption ratio 0.04 across seven aperture sizes.

would be produced by a larger single volume space with a blend of acoustic characteristics from the main and secondary volumes.

Although the general trends of these two DSE quantifiers are similar, the way in which T30/T15 and LDT/T10 quantify DSE is different, as shown in Fig. 3. The LDT/T10 values are higher than those for T30/T15, and show larger changes in DSE across configuration. The LDT/T10 values for the coupled configurations are higher than the single volume values. However, the T30/T15 values for aperture sizes $6.0 \%$ and $10.0 \%$ are lower than the single volume $(0 \%)$ value. The difference between the results of the two quantifiers suggests that LDT/T10 is able to quantify DSE more accurately and with a finer resolution than T30/T15.

The results from these two DSE quantifiers match well with a new quantity given in ISO 3382-2, the degree of curvature, which is used to determine the level of non-linearity of a decay curve. ${ }^{23}$ This metric is calculated by the ratio of T20 over T30 given as a percentage deviation from a perfectly exponential decay. Values higher than $10 \%$ are said to indicate a decay curve that significantly deviates from exponential decay. The four coupled configurations with the smallest aperture sizes $(0.5 \%, 1.0 \%, 1.5 \%$, and $3.0 \%)$ all have degrees of curvature higher than $10 \%$, indicating a significantly non-linear decay, which corresponds with the T30/ T15 and LDT/T10 quantifier results.

Two metrics, decay ratio and $\Delta L$, were also calculated for all the impulse responses filtered at the $1000 \mathrm{~Hz}$ octave band. These values were provided to the authors by Dr. Ning Xiang and his research group at Rensselaer Polytechnic Institute, by using their proprietary Bayesian analysis algorithm for room acoustics. The values calculated at receiver 1 are shown in Fig. 5. As found in previous studies, ${ }^{1,17}$ the decay ratio and $\Delta L$ data do not match well with the other DSE quantifiers. Only 5 of the 16 configurations that T30/ T15, LDT/T10, and the degree of curvature data indicate as being double slope are shown as double slope by decay ratio and $\Delta L$. Furthermore, decay ratio and $\Delta L$ values for the two largest aperture sizes $(6.0 \%$ and $10.0 \%)$ show significant 


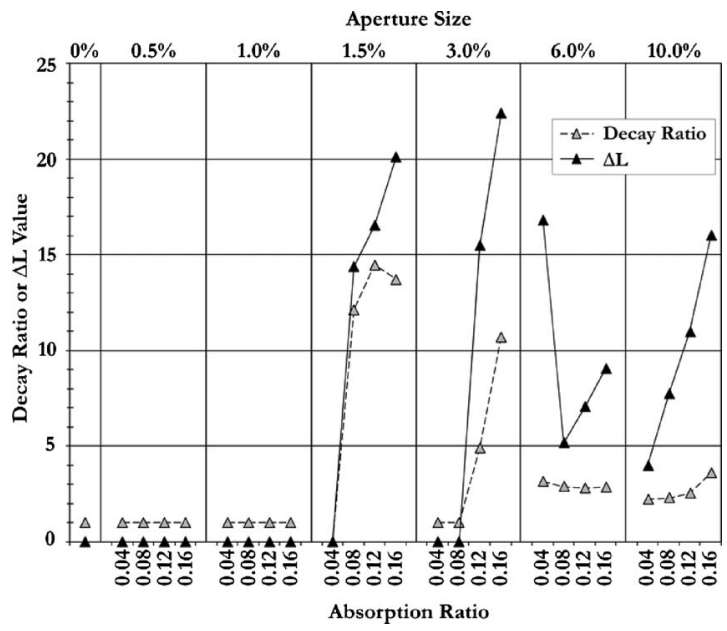

FIG. 5. Decay ratio and $\Delta L$ data for the 25 virtual hall configurations. Decay ratio equals 1 and $\Delta L$ equals 0 when only one slope has been detected by the Bayesian analysis.

DSE. By contrast the T30/T15 and LDT/T10 data for these aperture sizes showed single slope behavior, and the degree of curvature values were all less than $10 \%$. Theoretically, decay ratio and $\Delta L$ should most uniquely describe these double slope decay curves, but the authors have found that, in practice, the results are not always as expected. Further study is certainly required in calculating decay ratio and $\Delta L$ from Bayesian analysis of decay curves generated in realistic coupled volume spaces to determine the source of this inconsistency.

Among the DSE quantifiers reviewed in this paper, LDT/T10 is the one that produces the most comprehensible results while discerning the presence of DSE more finely than the other decay time ratios. Consequently, the remainder of this paper will use the LDT/T10 ratio as the primary quantifier for DSE.

\section{SUBJECTIVE ANALYSIS}

\section{A. Methodology}

In addition to the computational analysis, listener perception of the double slope decay profiles resulting from varying the architectural parameters of aperture size and absorption ratio was determined. Impulse responses from a subset of the virtual hall configurations were auralized. The results from the computational analysis were used to inform the configuration choice. In particular, configurations that produced disparate DSE results according to LDT/T10 were chosen, and configurations that produced similar DSE data were eliminated from the subjective testing subset. The data in Fig. 3 show similar DSE trends across absorption ratio within two groups of aperture sizes: group 1, consisting of aperture sizes $1.5 \%$ and $3.0 \%$, and group 2, consisting of sizes $6.0 \%$ and $10.0 \%$. One aperture size from each group was chosen for the subjective testing subset: aperture size $3.0 \%$ from group 1 and aperture size $10.0 \%$ from group 2 . The remaining configurations were retained for the subjective testing, resulting in 17 configurations, a combination of 4 absorption ratios and 4 aperture sizes $(0.5 \%, 1.0 \%, 3.0 \%$, and $10.0 \%$ ) plus the single volume case $(0 \%)$. To clarify the
TABLE V. Architectural parameter values of the 17 configurations used in the subjective testing with corresponding alpha-numeric labeling system.

\begin{tabular}{lccccc}
\hline \hline & & \multicolumn{4}{c}{ Absorption coefficient } \\
\cline { 3 - 6 } \multicolumn{1}{c}{$\begin{array}{c}\text { Aperture size } \\
(\%)\end{array}$} & & 0.04 & 0.08 & 0.12 & 0.16 \\
& & $a$ & $b$ & $c$ & $d$ \\
\hline 0 & 00 & $\ldots$ & $\ldots$ & $\ldots$ & $\ldots$ \\
0.5 & 1 & $1 a$ & $1 b$ & $1 c$ & $1 d$ \\
1.0 & 2 & $2 a$ & $2 b$ & $2 c$ & $2 d$ \\
3.0 & 4 & $4 a$ & $4 b$ & $4 c$ & $4 d$ \\
10.0 & 6 & $6 a$ & $6 b$ & $6 c$ & $6 d$ \\
\hline \hline
\end{tabular}

presentation of data from the subjective testing, these 17 configurations will be referred to using an alpha-numeric labeling system as given in Table $\mathrm{V}$.

ODEON (version 6.5) was used to produce auralizations of the 17 configurations. Binaural RIRs were convolved with an anechoic music signal provided with ODEON (Bach's Toccata et Fuga in D Minor), and a KEMAR head related transfer function (HRTF) was applied to each convolution. ${ }^{24}$ The Bach piece was chosen because of its wide pitch range, which excited the virtual hall at a variety of frequencies. Additionally, the $15 \mathrm{~s}$ sample had two runs of short notes played in quick succession with decreasing pitches, each concluding with a stop chord, allowing the listener to experience both stopped and running reverberation in the virtual space.

The convolution process resulted in 17 auralized sound tracks corresponding to the 17 configurations. The tracks were presented in a paired comparison test over electrostatic Sennheiser HE-60 headphones to 30 human test subjects. The paired comparison method is a form of psychometric scaling used to order stimuli along a given dimension (e.g., preference), based on a series of independent judgments made between all possible pairs of items. ${ }^{25}$ Subjects were asked to make a forced choice preference for each of the 136 pairs of auralized sound tracks. Specifically, for each paired comparison, the participant was told to "please listen to the audio samples and then answer the question: which track do you prefer?" The participant would then choose the first track or the second track using a radio button interface. Forced choice paired comparisons are used to create a binary response from the subject: Either track A is preferred or track $\mathrm{B}$ is preferred. Previous research by Parizet et al. ${ }^{26}$ examined the differences among several listening test methods; they showed that forced choice comparisons had the greatest range in scores, magnifying the size of the effect for subjective response to acoustic stimuli. Additionally, paired comparison tests were found to provide a higher discrimination power than tests in which subjects rate sounds individually.

In the current study, each subject was presented with the 136 aural stimuli in three sessions. Each session lasted approximately $1 \mathrm{~h}$, with a rest period of at least $2 \mathrm{~h}$ between sessions. The presentation order, both within pair and absolute order, was randomized for each participant to avoid bias error.

Subjects were members of the University of Nebraska community, ranging in age from 19 to 30 years, with an 
equal number of males and females. They all indicated that they had some experience listening to classical music in performing arts spaces. Only subjects with hearing thresholds lower than $25 \mathrm{~dB}$ hearing level (HL) across $125-8000 \mathrm{~Hz}$ in both ears were allowed to participate in the testing.

Prior to testing, the participants underwent a training session in which the general concepts of coupled volume systems and DSE were outlined. No indication was made regarding the types of acoustic conditions that should be preferred. Instead, the training module simply focused the participants on what they could expect to hear changing in the samples, and then the subjects were expected to base preference on their own internal scale that later could be mapped to external variables through subsequent multidimensional scaling (MDS) analysis. Upon completion of the training module, the subjects were presented with a graphical user interface (GUI), which directed them to choose their preferred track from each pair.

\section{B. Results}

The data from the subjective testing described above were analyzed using MDS analysis. This method is described below along with the results from the analysis. First, a discussion of the reliability of the subjective testing data is presented.

\section{Reliability of paired comparisons: Circular triads}

When more than three stimuli are presented to a subject, a circular triad (CT) response can occur wherein a valid preference hierarchy cannot be established. A paired comparison study resulting in a high rate of CTs suggests that subjects find it difficult to respond to the stimuli regarding the attribute in question (e.g., preference) ${ }^{25}$ This difficulty may be due to a high level of similarity among the stimuli, or the inappropriateness of the scale being used. Another possible cause for a high number of CTs is that the individual subject is unreliable or inconsistent in their responses. The consistency of individual subjects can be determined by Kendall's coefficient of consistency $(\xi)$, a metric used to determine if a participant has significantly fewer CTs than is expected by chance. ${ }^{27}$ Kendall and Babington-Smith showed that the maximum number of circular triads for an odd number of stimuli is given by

$$
\mathrm{CT}_{\max }=\frac{\left(N^{3}-N\right)}{24},
$$

where $N$ is the number of stimuli. The coefficient of consistency is calculated using

$$
\xi=1-\frac{d}{\mathrm{CT}_{\max }},
$$

where $d$ is the number of CTs produced by a subject. If a subject were to produce the maximum number of CTs, $\xi=0$, while with no CTs, $\xi=1$.

The probability of a given $\xi$ value based on the assumption that a subject's responses are completely random can be tested using a chi-square $\left(\chi^{2}\right)$ distribution. For a particular $d$, the $p$-value associated with the $\chi^{2}$ distribution indicates the probability of obtaining a number of CTs equal to or greater than $d$ based on chance, where a typical significance value of $p \leq 0.05$ is chosen for this type of test. ${ }^{28}$ The degree of consistency among all subjects can be represented by Kendall's coefficient of agreement $(u) .{ }^{27}$ The development of $u$ is similar to that of $\xi$, except that summations are made over the responses from all subjects. The coefficient ranges from 0 to 1 , with higher coefficient values indicating higher agreement among participants. A $\chi^{2}$ distribution can also be calculated for $u$, along with a corresponding probability. A significant $p$-value $(p \leq 0.05)$ for the coefficient is indicative of at least a minimal level of agreement among subjects. ${ }^{25}$

\section{Circular triad results}

A circular triad analysis was conducted on the paired comparison responses of all 30 subjects. The resulting coefficient of agreement was $u=0.0463 \quad(p=0.136)$, indicating that the agreement among subjects did not reach the minimum level. An investigation of the individual subjects' $\xi$ $p$-values revealed that the non-significance of $u$ can be attributed to the unreliability of certain subjects. Ten subjects produced a higher number of CTs than expected by chance ( $p$ $>0.05)$. However, the majority of participants $(66 \%)$ produced lower CT rates than expected by chance $(p \leq 0.05)$. The fact that most of the subjects were able to respond with a high coefficient of consistency suggests that individual participant unreliability, rather than a high similarity among stimuli or inappropriate scale usage, was the cause of the low agreement among subjects. Therefore, the "unreliable" subjects can be removed to increase the significance of $u .^{25}$

The ten subjects whose $\xi p$-value exceeded 0.05 were removed from the data set and a circular triad analysis was conducted once again. This modified subject set produced a $u=0.1117(p<0.05)$, indicating at least a minimum level of agreement among the participants. The data from the 20 "reliable" subjects were used in the MDS analysis discussed below.

\section{Multidimensional scaling}

MDS is a technique used to represent similarities between objects (or stimuli) as distances in a Euclidean space. $^{29}$ The more similar two objects are, the smaller the distance between them. While MDS assembles the Euclidean distance structure of the data, the researcher must assess the meaning for directions in that space, such as interpretation of the axes. ${ }^{29}$

MDS is an iterative process in which the accuracy of the statistical model is quantified by the output parameter stress, which is a numerical measure of the "badness-of-fit" between the current configuration and the input data. ${ }^{30}$ The MDS process is repeated until the difference between the calculated coordinates and the input data is minimized according to a predetermined stop criterion, such as a minimum stress level. Stress can also be used to determine the number of dimensions of the solution. In general, the stress level will decrease as the number of dimensions is increased. However, increasing the number of dimensions in the MDS solution can lead to visual obscurity and difficulty in inter- 


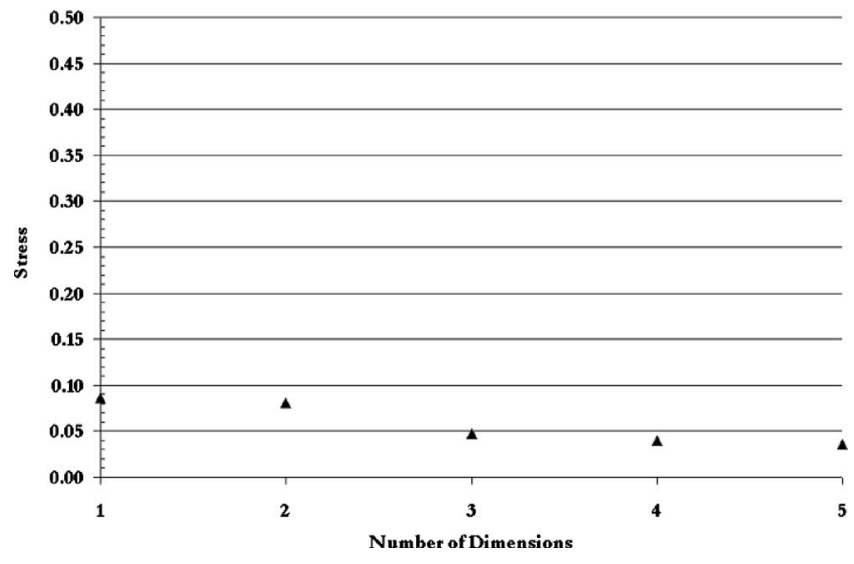

FIG. 6. A plot of number of dimensions versus stress. The stress value for dimension 1 is below 0.15 , with a small amount of change in stress as number of dimensions is increased, indicating dimension 1 is the correct dimension.

preting the coordinate axes; therefore, the Euclidean space with the fewest dimensions that best approximates the data is typically chosen as the final dimensional space for the solution. ${ }^{30}$ MDS is particularly useful in representing data describing stimuli whose categorization dimensions are not well understood. ${ }^{30}$ The limited body of research on subjective impression of DSE and the imprecise nature of most of the DSE quantifiers make MDS well suited for analyzing the psychoacoustic preference data to sound fields in coupled volume spaces.

\section{Multidimensional scaling results}

Each subject's data set was organized into a twodimensional $N \times N$ half matrix, where $N$ is the number of auralized virtual hall configurations $(N=17)$. Twenty matrices were generated, 1 for each of the 20 reliable subjects. Individual preference MDS analysis was conducted for oneto five-dimensional solutions. A true statistical method for determining which dimensionality yields the "correct" solution does not exist. However, since MDS is being used here as a descriptive model, considerations such as interpretability, ease of use, and stability can be used to determine an appropriate dimension value. ${ }^{29}$ Additionally, each dimensional solution yields a unique final stress value, which can be used to determine the solution that provides the best interpretation of the data. The stress values for each of the five solutions found through MDS analysis are shown in Fig. 6.

If the stress for dimension 1 is below 0.15 , then this strongly suggests that the one-dimensional solution is the most appropriate choice. ${ }^{29}$ The stress for dimension 1 in this case is 0.086 , indicating that the data should be interpreted in this dimension. Additionally, Fig. 6 shows little improvement in stress values with increasing number of dimensions, suggesting that the one-dimensional solution yields a good approximation of the data.

The MDS relative preference values, normalized to range from zero to six, are plotted against the LDT/T10 values in an $x-y$ scatter plot in Fig. 7. The configurations on the low end of the preference scale exhibit high levels of DSE (e.g., $1 a$ and $2 a$ ), while on the high end, the DSE levels are

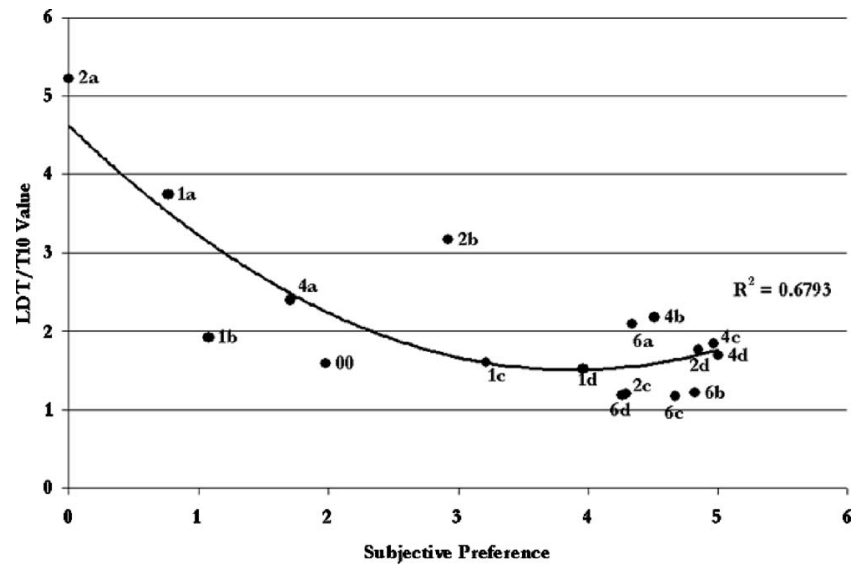

FIG. 7. Plot of relative subjective preference versus LDT/T10 data with a second-order polynomial trendline and correlation value. Higher subjective preference values indicate greater preference by listeners.

in the mid-range (e.g., $4 c$ and $4 d$ ). The lowest DSE values are located at the upper end of the middle of the preference scale. A second-order polynomial trendline corresponds with this increase in preference with decreasing DSE, and a slight peak in preference for medium levels of DSE. Also note that many of the coupled volume scenarios are preferred over the single main volume case (00).

The effect of variation in the virtual hall's architectural parameters on the MDS data set can be seen in Fig. 8, in which relative preference and DSE are plotted across configurations. The preference values are again normalized to range from zero to six so that they match the range of LDT/ T10 values. This normalization is acceptable since the MDS results have no inherent range (that is, the values are relative). The $y$-axis of Fig. 8 therefore has both the LDT/T10 and relative preference values shown together. In general, subjective preference increases with increasing absorption ratio, except for the aperture size level $6(10.0 \%)$ case. For this aperture size, preference peaks for absorption ratio $b$ (0.08). However, the changes in the MDS results and the LDT/T10 data for aperture level 6 are relatively small. Preference levels are largest for aperture level 4 (3.0\%). In general, the preference values are inversely proportional to DSE

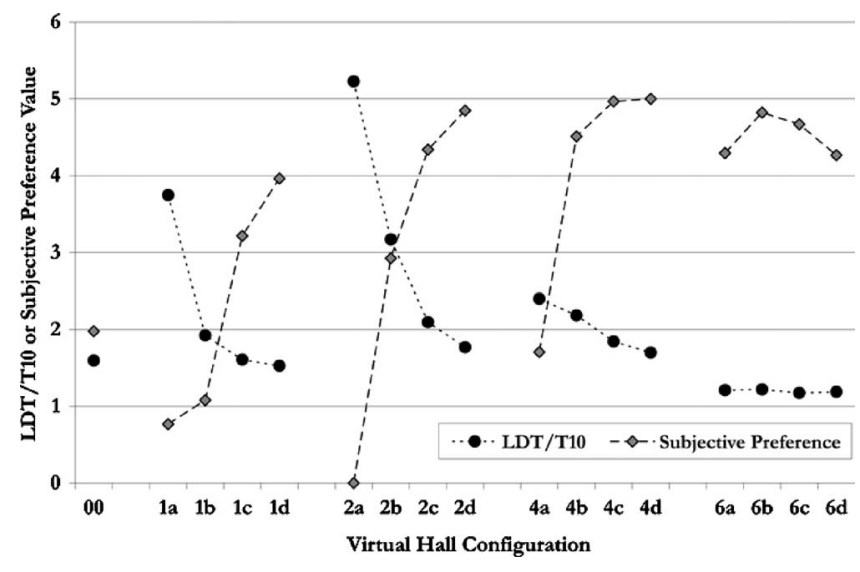

FIG. 8. Plot of LDT/T10 data versus relative MDS preference values for 17 configurations of virtual hall. Higher subjective preference values indicate greater preference by listeners. 
TABLE VI. Four levels of preference with the corresponding virtual hall configurations and architectural parameters

\begin{tabular}{ccccc}
\hline \hline $\begin{array}{c}\text { Preference } \\
\text { level }\end{array}$ & Configuration & $\begin{array}{c}\text { Volume ratio } \\
(\%)\end{array}$ & $\begin{array}{c}\text { Aperture size } \\
(\%)\end{array}$ & $\begin{array}{c}\text { Absorption } \\
\text { ratio }\end{array}$ \\
\hline 1 & $4 d$ & 47 & 3.0 & 0.16 \\
2 & $4 c$ & 47 & 3.0 & 0.12 \\
3 & $2 d$ & 47 & 1.0 & 0.16 \\
4 & $6 b$ & 47 & 10.0 & 0.08 \\
\hline \hline
\end{tabular}

level as quantified by LDT/T10. Overall, the data suggest that hall configurations producing low and medium levels of DSE are most preferred by listeners, with the highest preference occurring at medium sized aperture openings (3\% of the total main volume surface area) with larger absorption ratios (0.16). The lowest preference value is for configuration $2 a$ (aperture size 1.0\%: absorption ratio 0.04), which has the highest DSE level.

To further elucidate the effect of architectural variation on listener preference, the virtual hall configurations for the four highest levels of preference are listed in Table VI. The data from Table VI were used to develop the flow chart of design guidelines in Fig. 9(a). Each of the configurations in Table VI has a relatively large volume ratio (47\%). However, previous results have indicated that DSE decreases with decreasing volume ratio. ${ }^{1,10}$ Therefore, to generalize the design criteria for additional volume ratios, the other two architectural parameters have been adjusted accordingly in Figs. 9(b) and $9(\mathrm{c})$, which show suggested design guidelines for medium and small volume ratios.

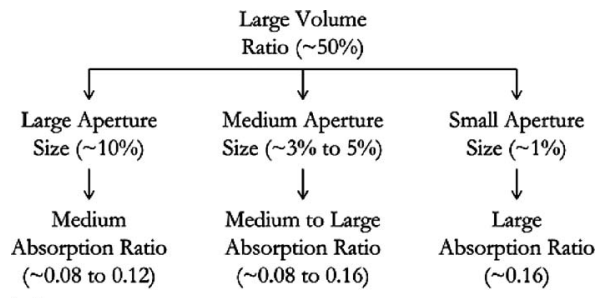

(a)

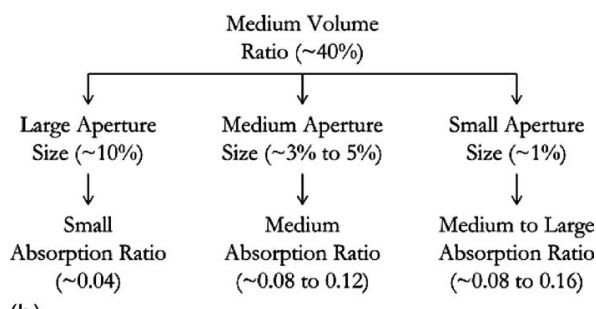

(b)

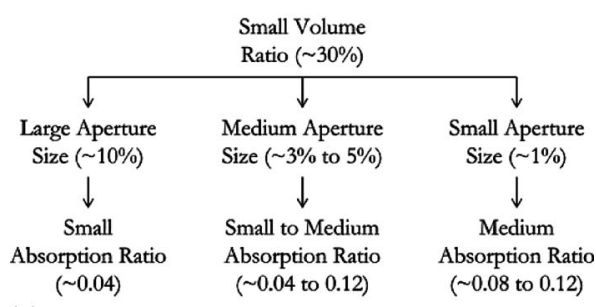

(c)

FIG. 9. Recommended design guideline flow charts for coupled volume concert halls with (a) large volume ratio, (b) medium volume ratio, and (c) small volume ratio.
The lowest level of preference from the subjective testing of the virtual hall was associated with the highest level of DSE, even lower than preference for the single main volume case (00). This level of DSE was produced by a configuration with a small aperture size $(0.5 \%)$ and a small absorption ratio (0.04). Therefore, these architectural parameter configurations, and others that would produce high levels of DSE as quantified by LDT/T10 values greater than 3, should be avoided in coupled volume design.

\section{CONCLUSIONS AND FUTURE WORK}

A virtual coupled volume concert hall was studied using computational modeling, in which two architectural parameters were varied: absorption ratio and aperture size. Four levels of absorption ratio and seven levels of aperture size were analyzed. Two decay ratios, T30/T15 and LDT/T10, were used to quantify the DSE, and the data from the analysis of the virtual hall show similar trends across the various configurations for both quantifiers. DSE decreases with increasing absorption ratio for each aperture size. DSE is highest at a specific aperture size for each absorption ratio. The high DSE aperture sizes were typically a small percentage of the total main volume surface area. Generally, LDT/T10 seemed to be a more discriminating quantifier of DSE than T30/T15. The metrics of decay ratio and $\Delta L$ were also calculated via a Bayesian analysis algorithm programed by Dr. Ning Xiang at Rensselaer Polytechnic Institute, but those results did not appear consistent with what was expected; in many of the cases tested, the presence of a double slope decay was not found. Further work is suggested in calculating decay ratio and $\Delta L$ from Bayesian analysis of decay curves generated in realistic coupled volume spaces to determine the source of this inconsistency.

This study has also provided a comprehensive analysis of preference related to realistic non-exponential decays with an appropriate subject pool size leading to significant results. Subjective testing was conducted to determine the effect on listener preference of varying aperture size and absorption ratio in the virtual hall. MDS analysis was used to determine the relative preference results for different virtual hall configurations. In general, listener preference was inversely proportional to DSE level, with the highest levels of preference occurring at low and medium levels of DSE. Many of these decay curves were preferred over the single volume case of the main hall alone, though. Based on the computational and subjective results, recommended design guidelines for coupled volume halls have been provided in Fig. 9.

Further work could focus on other coupled volume parameters, such as the location of coupling apertures, which was found to have some effect on DSE in a previous study. ${ }^{10}$ The distribution of absorption in the space warrants further investigation, as previous studies of single volume spaces have indicated that different absorption distributions cause significant changes in sound energy behavior in the space, such as a higher dependency on scattering coefficient values. ${ }^{31}$ The effect of surface scattering values could also be studied in more detail. For example, the scattering values of 
surfaces surrounding the coupling apertures may need to be higher than the ones used in this study to account for diffraction occurring at the aperture.

The realistic hall geometry studied in this research was based on the typical coupled volume hall, which is characterized by a rounded shoebox shape. Alternative geometries commonly seen in single volume halls, such as fan-shape and vineyard styles, could also be studied. Different modeling methods, such as physical scale modeling, radiative transfer models, ${ }^{32,33}$ diffusion models, ${ }^{34,35}$ and finite difference time-domain methods, ${ }^{36}$ could be used to make additional conclusions about the influence of architectural parameters on DSE and its subjective impression. Aspects of the subjective response to DSE could be further investigated as well. In particular, different music selections could be tested, or additional subjective preference tests could be run comparing double slope decays to a wider range of single slope decays. Furthermore, other subjective data collection methods, such as multiple level or continuous scale tests, might be used to gain additional insight into the preference levels for coupled volume spaces.

\section{ACKNOWLEDGMENTS}

The authors would like to thank Damian Doria of Artec Consultants, Inc. and Dr. Ning Xiang for their assistance with this project. This work was partially supported by an Acoustical Society of America Graduate Fellowship for Minorities and the Ford Foundation Diversity Fellowship Program.

${ }^{1}$ D. T. Bradley and L. M. Wang, "The effects of simple coupled volume geometry on the objective and subjective results from non-exponential decay," J. Acoust. Soc. Am. 118, 1480-1490 (2005).

${ }^{2}$ H. A. Davis, "Reverberation equations for two adjacent rooms connected by an incompletely soundproof partition," Philos. Mag. 50, 75-80 (1925).

${ }^{3}$ L. Cremer and H. A. Muller, Principles and Applications of Room Acoustics (Applied Science, New York, 1982), Vol. 1.

${ }^{4}$ A. Munro and R. Johnson, "Calculating reverberation time in coupled rooms," Workshop on Design of Concert Chambers, Tanglewood '99 (Concert Hall Research Group, Summer Institute, Lenox, MA, 1999).

${ }^{5}$ N. Xiang and P. M. Goggans, "Evaluation of decay times in coupled spaces: Bayesian parameter estimation," J. Acoust. Soc. Am. 110, 14151420 (2001).

${ }^{6} \mathrm{~N}$. Xiang and P. M. Goggans, "Evaluation of decay times in coupled spaces: Bayesian decay model selection," J. Acoust. Soc. Am. 113, 26852697 (2003).

C. F. Eyring, "Conditions under which residual sound in reverberant rooms may have more than one rate of decay," J. Soc. of Motion Picture Engineers 15, 528-538 (1930).

${ }^{8}$ C. F. Eyring, "Reverberation time measurements in coupled rooms," J. Acoust. Soc. Am. 3, 181-206 (1931).

${ }^{9}$ L. Nijs, G. Jansens, and M. van der Voorden, "The prediction of sound pressure levels in coupled rooms using ray-tracing models," Inter-Noise 98, Christchurch, New Zealand (1998).

${ }^{10} \mathrm{~B}$. Harrison and G. Madaras, "Computer modeling and prediction in the design of coupled volumes for a 1000-seat concert hall at Goshen College, Indiana," J. Acoust. Soc. Am. 109, 2388(A) (2001).

${ }^{11}$ J. E. Summers, R. R. Torres, and Y. Shimizu, "Statistical-acoustics models of energy decay in systems of coupled rooms and their relation to geometrical acoustics," J. Acoust. Soc. Am. 116, 958-969 (2004).

${ }^{12}$ J. E. Summers, R. R. Torres, and Y. Shimizu, "Adapting a randomized beam-axis-tracing algorithm to modeling of coupled rooms via late-part ray tracing," J. Acoust. Soc. Am. 118, 1491-1502 (2005).

${ }^{13}$ M. Ermann, "Coupled volumes: Aperture size and the double-sloped decay of concert halls," Build. Acoust. 12, 1-14 (2005).

${ }^{14}$ D. T. Bradley and L. M. Wang, "Comparison of measured and computermodeled objective parameters for an existing coupled volume concert hall," Build. Acoust. 14, 79-90 (2007).

${ }^{15}$ ISO 3382-1, "Acoustics-Measurement of Room Acoustic ParametersPart 1: Performance Spaces," 2009.

${ }^{16}$ N. Xiang, P. M. Goggans, T. Jasa, and M. Kleiner, "Evaluation of decay times in coupled spaces: reliability analysis of Bayesian decay time estimation," J. Acoust. Soc. Am. 117, 3707-3715 (2005).

${ }^{17}$ D. T. Bradley and L. M. Wang, "Quantifying the double slope effect in coupled volume room systems," Build. Acoust. 16, 105-123 (2009).

${ }^{18}$ B. S. Atal, M. R. Schroeder, and G. M. Sessler, "Subjective reverberation time and its relation to sound decay," Proceedings of the Fifth International Congress on Acoustics, Liege, Belgium (1965), Paper No. G32.

${ }^{19}$ D. Picard, "Audibility of non-exponential reverberation decays," Rapport de Stage D’Option Scientifique Rensselear Polytechnic Institute, 2003.

${ }^{20}$ M. Ermann, "Double sloped decay: Subjective listening test to determine perceptibility and preference," Build. Acoust. 14, 91-107 (2007).

${ }^{21}$ C. L. Christensen, ODEON room acoustics program, User Manual v6.5, Industrial, Auditorium and Combined Editions, Odeon A/S, Denmark, 2003.

${ }^{22}$ C. L. Christensen, "ODEON-A design tool for auditorium acoustics, noise control and loudspeaker systems," Proceedings of the Institute of Acoustics: Reproduced Sound 17, Measuring, Modeling, or Muddling, Vol. 23, pp. 137-144 (2004).

${ }^{23}$ ISO 3382-2, "Acoustics-Measurement of Room Acoustic ParametersPart 2: Reverberation Time in Ordinary Rooms," 2008.

${ }^{24}$ M. D. Burkhard and R. M. Sachs, "Anthropometric manikin for acoustic research,” J. Acoust. Soc. Am. 58, 214-222 (1975).

${ }^{25}$ H. A. David, The Method of Paired Comparisons (Oxford University Press, New York, 1988).

${ }^{26}$ E. Parizet, N. Hamzaoui, and G. Sabatie, "Comparison of some listening test methods: a case study," Acta Acust. 91, 356-364 (2005).

${ }^{27}$ M. G. Kendall and B. Babington-Smith, "On the method of paired comparisons," Biometrics 31, 43-62 (1939).

${ }^{28}$ G. Knezek, S. Wallace, and P. Dunn Rankin, "Accuracy of Kendall's chisquare approximation to circular triad distributions," Psychometrika 63, 23-34 (1998).

${ }^{29}$ J. B. Kruskal and M. Wish, Multidimensional Scaling (Sage, Newbury Park, CA, 1978).

${ }^{30}$ S. S. Schiffman, M. L. Reynolds, and F. W. Young, Introduction to Multidimensional Scaling (Academic, New York, 1981).

${ }^{31}$ L. M. Wang and J. Rathsam, "The influence of absorption factors on the sensitivity of a virtual room's sound field to scattering coefficients," Appl. Acoust. 69, 1249-1257 (2008)

${ }^{32}$ E. Nosal, M. Hodgson, and I. Ashdown, "Improved algorithms and methods for room sound-field prediction by acoustical radiosity in arbitrary polyhedral rooms," J. Acoust. Soc. Am. 116, 970-980 (2004).

${ }^{33}$ M. Hodgson and E. Nosal, "Experimental evaluation of radiosity for room sound-field prediction," J. Acoust. Soc. Am. 120, 808-819 (2006).

${ }^{34}$ A. Billon, V. Valeau, A. Sakout, and J. Picaut, "On the use of a diffusion model for acoustically coupled rooms," J. Acoust. Soc. Am. 120, 20432054 (2006)

${ }^{35}$ Y. Jing and N. Xiang, "Visualizations of sound energy across coupled rooms using a diffusion equation model," J. Acoust. Soc. Am. 124, EL360-EL365 (2008)

${ }^{36}$ J. M. Botts and N. Xiang, "An application of the finite difference timedomain method to the study of coupled volumes," J. Acoust. Soc. Am. 125, 2735(A) (2009). 